\title{
The Impact of the COVID-19 Pandemic on Radiation Therapy Delivery for Pediatric Patients: Trainee Perspective and Practical Challenges
}

\author{
Nikol Mladkova-Suchy, Joshua Palmer
}

Department of Radiation Oncology, Arthur G. James Cancer Hospital \& Richard J. Solove Research Institute, United States.

\begin{abstract}
The COVID-19 global pandemic represents a unique challenge affecting all aspects of current life including the delivery of healthcare around the globe. Radiation treatment is an integral part in the management of many pediatric malignancies, and the aim is to provide our institutional experience and trainee perspective on the delivery of radiation treatment during this era to facilitate further discussion regarding the practical impact of the pandemic on the treatment of childhood cancers and trainee education. Overall, the effect of the virus on the population of children with malignancies and its possible impact on their overall outcome is uncertain. The impact on trainee education is inevitable but can be mitigated in the context of adequate personal safety measures and online education. The authors strongly advocate for data sharing among facilities to determine the optimal safety measures that decrease the likelihood of COVID-19 transmission yet do not compromise the delivery of radiation treatment to children.
\end{abstract}

Keywords: COVID-19-radiation treatment-radiotherapy-pediatric cancer

Asian Pac J Cancer Care, 5 (Suppl 1), 229-230

\section{Introduction}

The coronavirus disease 2019 (COVID-19) emerged as a new global infectious threat in December 2019 following reports of a new severe acute respiratory syndrome in the city of Wuhan in the Chinese Province of Hubei [1]. The disease turned out to be caused by SARS-CoV-2, a novel coronavirus, and its rapid spread around the globe lead the World Health Organization to declare the virus outbreak a pandemic on 3/11/2020. In the state of Ohio, the first confirmed cases of COVID19 infection were reported $3 / 10 / 2020$. As of July $7^{\text {th }}, 2020$, Ohio has recorded a total of 58,904 cumulative confirmed COVID19 infection cases [2]. The governor of Ohio issued a stay at home order on $3 / 23 / 2020$ which remained in effect until 5/29/2020.

\section{Institutional Response}

The Ohio State University health system swiftly responded to the pandemic by implementing several personal safety measures for providers including but not limited to: Minimal physical contact with patients
Submission Date: 07/10/2020Ａcceptance Date: 08/13/2020

Corresponding Author:

Dr. Nikol Mladkova-Suchy

Department of Radiation Oncology, Arthur G. James Cancer Hospital \& Richard J. Solove Research Institute, United States.

Email: Nikol.mladkova@osumc.edu 
oncology consultations were scheduled in a timely fashion for an in-person visit. Patients who completed treatment prior and required a follow-up visit were typically scheduled for a telehealth appointment with their radiation oncologist. If it was deemed that those patients may require additional treatment, the patient was then scheduled for an in-person visit with a CT simulation typically coordinated on the same day to minimize exposure risk.

From with a trainee perspective, all educational activities were moved online including lectures and workshops. This did not impact the number or quality of educational activities.

\section{Radiation Treatment Delivery to Pediatric Patients}

All patients that required radiation treatment had to undergo COVID-19 test to be negative prior to initiating radiation, including pediatric patients. If the patient was deemed suspected for the infection while on treatment before the test came back with a definitive result, additional safety measures were taken for treatment delivery: they were treated as though they were positive with gowns, gloves, face shields and N95 masks, and those patients were typically treated at the end of the day as the radiation treatment room required additional clean-up.

The delivery of radiation treatment to pediatric patients was not compromised by the COVID-19 pandemic including to those patients who require anesthesia. However, the patients were experiencing differences in the usual approach to care delivery including the absence of both of their parents during their consultations and on treatment visits, as only 1 adult was allowed to accompany them. Many parents who could not be present for the visits were connected through audiovisual technology such as video conferencing and were included in the conversations regarding the necessity of radiation, treatment plan discussions and were given the opportunity to ask questions. In spite of this, some voiced their understandable frustration and disappointment that they cannot be personally present for those visits.

All pediatric patients were required to wear surgical masks during the visits including very young children. As a personal observation, those were typically very well tolerated even by the youngest patients. The communication with their other providers was not interrupted, as those were reachable by phone or e-mail, and many cases were discussed during weekly multidisciplinary conference which took place online.

\section{Radiation Oncology Trainee - educational impact}

As a trainee in radiation oncology, Dr. Mladkova was involved in seeing all new pediatric patients and all known patients who required treatment. Due to the effort to minimize the risk of exposure for patients by seeing multiple providers, Dr. Mladkova was not a part of all on-treatment visits for every single patient on treatment, but their course was always discussed with me if she was not present for their on-treatment visit. The major difference compared to the standard set up in the clinic was that routine follow-up visits took place over the phone with the attending physician. This perhaps created the biggest educational change compared to pre-COVID19 era.

To counteract this fact, our department organizes a weekly pediatric team meeting during which both new and known cases are discussed and this provides an excellent additional opportunity for trainee education: many of the patients who had telehealth appointments were reviewed during this meeting and this provided an additional educational background to the trainee.

In conclusion, very little is known with respect to COVID-19 impact on children with cancer. It is not possible yet to say with certainty that COVID-19 had not affected the course of care for children with cancer that required radiation treatment, as changes in the referral and surveillance pattern need to be explored to determine this with adequate confidence, along with long-term follow-up data. Radiation treatment at our institution was delivered in a standard albeit modified fashion mainly impacting the character and nature of in-person visits. Technology allowed for participation of additional family members who were not allowed to be a part of clinic visits due to COVID-19 visitor policy, and for the continuation of trainee education. Further collaborative data is needed to determine the best approach with respect to treatment delivery during a pandemic.

\section{References}

1. Zhu N, Zhang D, Wang W, Li X, Yang B, Song J, Zhao X, Huang B, Shi W, Lu R, Niu P, Zhan F, Ma X, Wang D, $\mathrm{Xu}$ W, Wu G, Gao GF, Tan W. A Novel Coronavirus from Patients with Pneumonia in China, 2019. New England Journal of Medicine. 202002 20;382(8):727-733. https:// doi.org/10.1056/nejmoa2001017

2. Dong E, Du H, Gardner L. An interactive web-based dashboard to track COVID-19 in real time. The Lancet Infectious Diseases. 2020 05;20(5):533-534. https://doi.org/10.1016/ s1473-3099(20)30120-1

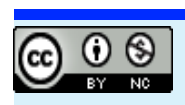

This work is licensed under a Creative Commons AttributionNon Commercial 4.0 International License. 